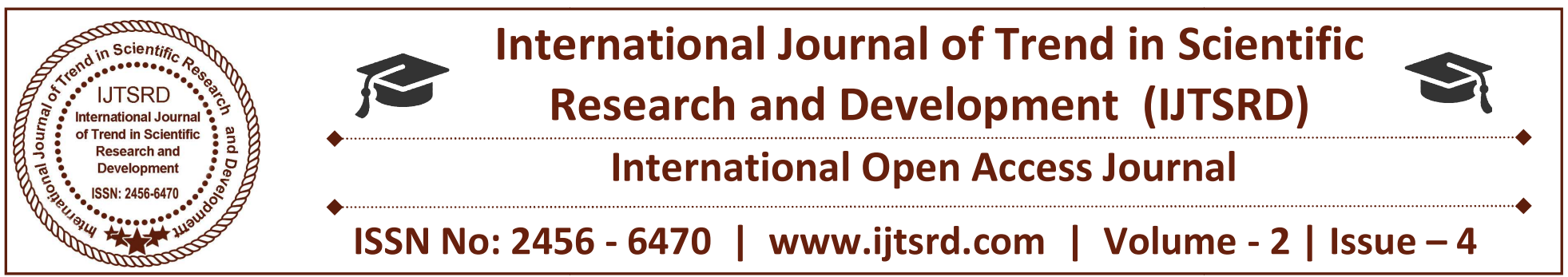

\title{
Bio-Toxicity of Materials at the Nanoscale Level
}

\author{
K. Vinoth Kumar \\ Research Associate, Department of Environmental Sciences \\ Agricultural University, Coimbatore, Tamil Nadu, India
}

\section{ABSTRACT}

Over the past decade, nanomaterials have been the subject of enormous interest. Nanomaterials notable for their extremely small feature size, have the potential for wide-ranging of applications. Nanomaterial is a field which takes a materials science- based approach to nanotechnology. It studies materials with morphological features on the nanoscale and especially those which have special properties from their nanoscale dimensions. The particle size and surface area are important material characteristics from a toxicological perspective. In this paper, the definitions, types, transformation and toxicity mechanisms of nanomaterials are summarized. Subsequently, the toxicity potential of nanomaterials on plants and microbe also detailed.

Keywords: Nanomaterials, Toxicity, Plant, Microbes

\section{INTRODUCTION}

Nanomaterials are engineered structures with at least one dimension of $100 \mathrm{~nm}$ or less. These materials are increasingly being used for commercial purposes such as fillers, catalysts, semiconductors, cosmetics, microelectronics, and drug carriers. Materials in this size range may approach the length scale at which some specific physical or chemical interactions with their environment can occur. As a result, their properties differ substantially from those bulk materials of the same composition, allowing them to perform exceptional feats of conductivity, reactivity and optical sensitivity. Possible undesirable results of these capabilities are harmful interactions with biological systems and the environment, with the potential to generate toxicity (Nel et al., 2006).

\section{Engineered Nanoparticles (ENP)}

The engineered nanoparticles are take place as a result of manmade industrial processes and it is also called "manufactured nanoparticles" having a specific properties or a specific composition. These particles can be made of single elements like carbon or silver or a mixture of elements / molecules (Monica and Cremonini, 2009). These engineered nanoparticles have received a more attention for their positive impact in improving many sectors of economy, including / $/$ consumer products, pharmaceutics, cosmetics, transportation, energy and agriculture etc., and are being increasingly produced for a wide range of applications within industry (Novack and Bucheli, 2007). Because of their widespread use in consumer products it is expected that nanoparticles will find their way into aquatic, terrestrial and atmosphere environments, where their fate and behaviour are largely unknown. Therefore, organisms and especially those that interact strongly with their immediate environments are expected to be affected as a result to their exposure to nanoparticles. The properties of engineered nanoparticles are attributable to their size (surface area and size distribution), chemical composition (purity, crystallinity, electronic properties), surface structure (surface reactivity, surface groups, inorganic or organic coatings), solubility, shape and aggregation. Although impressive from a physicochemical viewpoint, the novel properties of nanoparticles raise concerns about the adverse effects on biological systems ( $\mathrm{Nel}$ et al., 2006). 


\section{Types of engineered nanoparticles}

Engineered nanoparticles can be grouped into four types: a) carbon based materials, usually including fullerene, Single Walled Carbon Nanotube (SWCNT) and Multiwalled Carbon Nanotubes (MWCNT), b) metal based materials such as quantum dots, nanogold, nanozinc, nanoaluminum and nanoscales metal oxides like $\mathrm{TiO}_{2}, \mathrm{ZnO}$ and $\mathrm{Al}_{2} \mathrm{O}_{3}$, c) dendrimers which are nano-sized polymers built from branched units, capable of being tailored to perform specific chemical function and d) composites which combine nanoparticles with other nanoparticles or with larger bulk-type materials (Lin and Xing, 2007) and present different morphologies such as spheres, tubes, rods and prisms (Yu-Nam and Lead, 2008).

\section{Transformation in the environment}

The growing production and use of nanomaterials will inevitably leads to their accumulation in environment, which can have major implications for living organisms and environment. Nanoparticles may have an impact in the environment naviz., (i) a direct effect on biota, (ii) changes in the bioavailability of toxins or nutrients, (iii) indirect effects resulting from their interaction with natural organic compounds and (iv) changes in the environmental microstructures (Simonet and Valcarcel, 2009).

\section{Toxicity mechanism}

The particle size and surface area are important material characteristics from a toxicological perspective. As the size of the particle decrease, its surface area increase and also allow a greater proportion of its atoms or molecules to be displayed on the surface rather than the interior of the material (Fig. 1). The increase in surface area determines the potential number of reactive groups on the particles surface (Nel et al., 2006). The change in the physicochemical and structural properties of engineered nanoparticles with a decrease in size could be responsible for a number of material interactions that could lead to toxicological effects. For instance, shrinkage in size may create discontinuous crystal planes that increase the number of structural defects as well as disrupt the well structural electronic configuration of the material, so as to give rise to altered electronic properties (Oberdorster et al., 2005). This could establish specific surface groups that could function as reactive sites. The extent of these changes and their importance strongly depend on the chemical composition of the material. Surface groups can make nanoparticles hydrophilic or hydrophobic, lipophilic or lithopobic and catalytically active or passive. An example of how those surface properties can lead to toxicity is the interaction of electron donor or acceptor active site (chemically or physically activated) with molecular dioxygen $\left(\mathrm{O}_{2}\right)$. Electron capture can lead to the formation of the superoxide radical $\left(\mathrm{O}_{2} *\right)$, which through dismutation or Fenton chemistry can generate additional Reactive Oxygen Species (ROS).

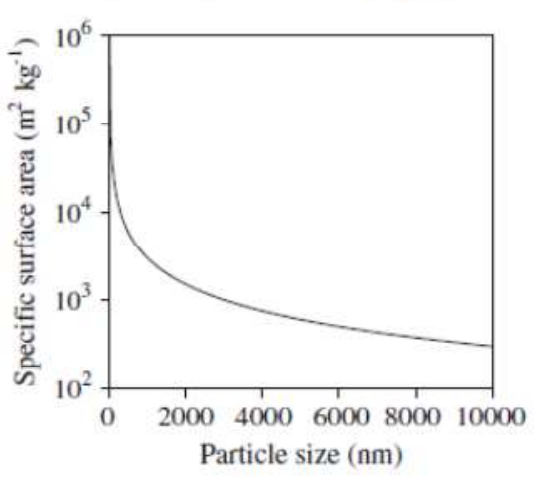

Fig 1: Relationship between specific surface area $\left(\mathrm{m}^{2} \mathrm{~kg}^{-1}\right)$ of a spherical particles and its size (diameter in nm) with a density of $1000 \mathrm{~kg} \mathrm{~m}^{-3}$

Single-component materials as well as presence of transition metals on the surface can participate in the formation of such active sites. For instance, an ultrafine particle contains transition metals (e.g. Fe and Vanadium) and is also coated with redox-cycling organic chemicals (quinines), whereas carbon nanotubes contain metal impurities that can amplify chemical changes in the nanoparticle environment. Thus, several nanoparticles characteristics can culminate in ROS generation, which is currently the best-developed paradigm for nanoparticles toxicity (Shvedova et al., 2003). Other nanopaticle properties such as shape, aggregation, surface coating and solubility may also affect the addressed specific physicochemical and transport properties, with the possibility of negating or amplifying the size effects.

\section{Effects on plant systems}

Higher plants strongly interact with their atmospheric and terrestrial environments and are expected to be affected as a result of their exposure to nanoparticles. Studies on the toxicity of nanomaterials are still emerging and basically evidence several negative effects on growth and development of plants. These 
results are based on tests suggested and encoded by USEPA (1996) that consider studies on seed germination, root elongation, often accompanied by other evaluations on biomass changes and anatomicalhistological studies, useful to evidence in-situ symptoms of possible toxicity.

Limited studies were reported both positive and negative effects of nanoparticles in higher plants. Lin and Xing (2007) analyzed phytotoxicity of five types of multiwalled nanoparticles at the level of seed germination and root growth in six higher plant species (Raphanus sativus, Brassica napus, Lolium multiflorum, Lactuca sativa, Zea mays and Cucumis sativus). Seed germination was not affected except for the inhibition of nanoscale zinc on Lolium multiflorum and nanoscale zinc oxide on Zea mays. Inhibition of root growth varied greatly among nanoparticles and plants and it is partially correlated to nanoparticles concentration. Yang and Watts (2005) investigated the phytotoxicity of nano-scale alumina powder with or without phenanthrene coating on five plant species (Zea mays, Cucumis sativus, Glycine max, Brassica oleracea and Daucus carota). The inhibition of root elongation was observed in all plant species with uncoated alumina nanopaticles.

Lee et al. (2008) analyzed toxicity and bioavailability of copper nanoparticles to the plants Phaseolus radiatus and Triticum aestivum employing plant agar test as growth substrate for homogeneous exposure of nanoparticles. Plant agar, which is soft gel, allows dispersion of nanoparticles, hardly water soluble, avoiding their precipitation. The growth rates of both plants were inhibited and as result of exposure to nanoparticles and the seedling lengths of tested species were negatively related to the exposure concentration of nanoparticles. Bioaccumulation is concentration dependent and the contents of nanoparticles in plant tissues increased with increasing nanoparticles concentration in growth media.

Racuciu and Creanga (2007) investigated the influence of magnetic nanoparticles coated with tetramethyl ammonium hydroxide on the growth of Zea mays plant in early onto genetic stages. The authors affirmed that water based ferrofluid addition in culture medium represents a source of iron. The iron based nanoparticles may have not only a chemical but also a magnetic influence on the enzymatic structures implied at different stages of photosynthesis. Small concentrations of aqueous ferrofluid solution added in culture medium had a stimulating effect on the growth of the plantlets while the enhanced concentration of aqueous ferrofluid solution induced an inhibitory effect. Battke et al. (2008) analyzed the uptake of palladium (Pd) by barley (Hordeum vulgare) and the behaviour of $\mathrm{Pd}$ nanoparticles in nutrient solutions used to grow plants. Smaller and larger $\mathrm{Pd}$ particles were comparatively assessed and the results showed that $\mathrm{Pd}$ uptake viz., the roots, depends on its particle diameter. Smaller Pd particles cause stress effects in leaves at low concentration in nutrient solution.

The mixture of nanoscale $\mathrm{SiO}_{2}$ and $\mathrm{TiO}_{2}$ could increase nitrate reductase in soybean (Glysine max), enhance its abilities of absorbing and utilizing water and fertilizer, stimulate its antioxidants system and apparently hasten its germination and growth ( $\mathrm{Lu}$ et al., 2002). Zhang et al. (2005) analyzed the effects of nano- $\mathrm{TiO}_{2}$ and non nano- $\mathrm{TiO}_{2}$ on the germination and growth of naturally aged seeds of spinach (Spinacia oleracea) by measuring the germination rate and vigor index. An increase of these indexes was observed at 0.25 to 4.0 per cent nano- $\mathrm{TiO}_{2}$ treatments. During the growth stage the plant dry weight, chlorophyll fiformation, ribulose bisphosphate carboxylase/ oxygenase activity and the photosynthetic rate were increased. The best results were found in 2.5 per cent of nano- $\mathrm{TiO}_{2}$. These results evidenced that the physiological effects were related to the nanometer-size particles. Nanoparticles are able to produce the anatomical changes in plants. Lin and Xing (2008) analyzed the effect of $\mathrm{ZnO}$ nanoparticles on root anatomical structure of ryegrass. Shrank morphology of the root tips of ryegrass was observed due to the influence of $\mathrm{ZnO}$ nanoparticles.

\section{Effects on soil biological communities}

Nanoparticles are introduced into the soil as a result of a number of human activities, including deliberate releases viz., soil and water remediation technologies, potential agricultural uses (eg. Fertilizer) and unintentional release viz., air, water and sewage applied to the lands (Shah and Belozerova, 2009). Microorganisms play a very important role in maintaining the soil health, ecosystem functions and production. Many nanoparticles have already been reported to have anti-microbial properties and thus directly affect microorganisms. Microbial toxicity has been reported for titanium dioxide and fullerene nanoparticles by Lovern and Klaper (2006). Soni and Bondi (2004) elucidated the mechanisms of action of 
how silver nanoparticles damaged bacterial cells. They reported that silver nanoparticles damaged and pitted the cell wall of Escherichia coli and accumulated in the cell wall, leading to increased cell permeability and ultimately cell death. Sabila et al., 2006, reported that nanoparticles of zinc oxide and magnesium oxide have been shown to be efficient in killing microorganisms and are used as preservatives in food. Iron and copper based nanoparticles could be presumed to react with peroxides present in the environment generating free radicals. These radicals are known to be highly toxic to microorganisms.

\section{CONCLUSION}

Nanotoxicity, an emerging concept, is receiving increasing attention with the fast development of nanotechnology. The main characteristic of engineered nanoparticles is their size $(<100 \mathrm{~nm})$, which falls in the transitional zone between individual atoms or molecule and the corresponding bulk materials. This can modify the physicochemical properties of the material as well as create the opportunity for increased uptake and interaction with biological systems. This combination effect can generate adverse biological effects in living cells that would not otherwise be possible with the same material in large form. Other properties, such as high specific surface area, abundant reactive sites on the surface as a consequence of a large fraction of atoms located on the exterior rather than in the interior of engineered nanoparticles, as well as their mobility, could potentially lead to unexpected health and environmental hazards. Therefore, organisms and especially those that interact strongly with their immediate environments are expected to be affected as a result of their exposure to engineered nanoparticles.

\section{REFERENCES}

1. Battke, F., K. Leopold, M. Maier, U. Schidhalter and M. Schuster. 2008. Palladium exposure of barley uptake and effects. Plant Bio., 10: 272-276.

2. Lee, W. M., Y.J. An, H. Yoon and H. S. Kwbon. 2008. Toxicity and bioavailability of copper nanoparticles to the terrestrial plants mung bean (Phaseolus radiatus) and wheat (Triticum aestrivum) plant agar test for water-insoluble nanoparticles. Environ. Toxicol. Chem., 27: 19151921.
3. Lin, D and B. Xing. 2007. Phytotoxicity of nanoparticles: Inhibition of seed germination and root growth. Environ. Pollut., 150: 243-250.

4. Lin, D and B. Xing. 2008. Root uptake and phytoxoxicity of $\mathrm{ZnO}$ nanoparticles. Environ. Sci. Technol., 42: 5580-5585.

5. Lovern, S. B and R. Klaper. 2006. Daphnia magna mortality when exposed to titanium dioxide and fullerene (C60) nanoparticles. Environ. Toxicol. Chem., 25: 1132-1137.

6. Lu, C.M., C.Y. Zhang, J.Q. Wen, G.R. Wu and M.X. Tao. 2002. Research of the effect of nanometer materials on germination and growth enhancement of Glysine max and its mechanism. Soybean Sci., 21: 168-172.

7. Monica, R.C and R. Cremonini. 2009. Nanoparticles and higher plants. Caryologia, 62 (2): 161-165.

8. Nel, A., T. Xia, L. Madler and N. Li. 2006. Toxicity potential of materials at the nanolevel. Science, 311: 622-627.

9. Nowack, B and T.D. Bucheli. 2007. Occurrence, behavior and effects of nanoparticles in the environment. Environ. Pollut., 150: 5-22.

10. Oberdorster, G., E. Oberdorster and J. Oberdorster. 2005. Nanotoxicology: An emerging discipline evolving from studies of ultrafine particles. Environ. Health Perspect, 113: 823-839.

11. Racuciu, $M$ and D. E. Creanga. 2007. TMA-OH coated magnetic nanoparticles internalized in vegetal tissues. Romanian J. Phys., 52: 395-395.

12. Sabila. A.M., R. Nishi, B. Raymond, E.A. Marques, U. G. Lopes, L. Touqui and M.C. Plotowski. 2006. Implications of oxidative stress in the cytotoxicity of Pseudomonas aeruginosa. Microbes Infect., 2: 450-459.

13. Shah, V and I. Belozerova. 2009. Influence of metal nanoparticles on the soil microbial community and germination of lettuce seeds. Waste Air Soil Pollut., 197: 143-148.

14. Shvedova, A.A., V. Castranova, E.R. Kisin, D.S. Berry, A.R. Murray, V.Z. Gandelsman, A. Maynard and P. Baron. 2003. Exposure to carbon nanotube material: Assessment of nanotube cytotoxicity using human keratinocyte cells. $J$. Toxicol. Envrion. Health, 66: 1909-1926. 
15. Simonet, B.M and M. Valcarcel. 2009. Monitoring nanoparticles in the environment. Anal. Bioanal. Chem., 393: 17-21.

16. Soni, I and S.B. Bondi. 2004. Silver nanoparticles as antimicrobial agent: A case study on E.coli as a model for gram-negative bacteria. J. Colloid Interface Sci., 275: 1770-1782.

17. USEPA (United States Environmental Protection Agency). 1996. Seed Germination and Root Elongation Toxicity Test. Ecological effects test guidelines (OPPTS 850.4200).

18. Yang, L and D.J. Watts. 2005. Particle surface characteristics may play an important role in phytotoxicity of alumina nanoparticles. Toxicol. Lett., 158: 122-132.

19. Yu-Nam, Y and R. Lead. 2008. Manufactured nanoparticles: An overview of their chemistry, interactions and potential environmental implications. Sci. Total Environ., 400: 396-414.

20. Zhang, L., F. Hong, S. Lu and C. Liu. 2005. Effect of nano- $\mathrm{TiO}_{2}$ on strength of naturally aged seeds and growth of Spinach. Bio.Trace Elem. Res., 105: 83-91. 